\title{
Using maximum-likelihood adaptive methods to estimate difference thresholds and points of subjective equality
}

\author{
ROBERT A. POKORNY \\ U.S. Air Force Research Laboratory, Brooks Air Force Base, Texas
}

\begin{abstract}
In studying perception, psychologists must sometimes estimate perceivers' difference thresholds and points of subjective equality (PSEs). To quickly estimate a threshold, one adaptive procedure that has been claimed to find thresholds with maximal efficiency is Pentland's Best PEST. Descriptions of Best PEST report its implementation in the finding of one point along the perceiver's psychometric function. To find a difference threshold (also called difference limen, or DL) and the PSE, two thresholds must be found (as two points are required to estimate the slope and intercept of the perceiver's psychometric function). This paper describes how to modify Best PEST so as to estimate any threshold along the perceiver's psychometric function and reports the results of simulations in which both the DL and the PSE are estimated.
\end{abstract}

Some psychological questions require that psychologists measure (1) how perceivers discriminate one signal from another and (2) how perceivers match one signal to another. Traditionally, how perceivers discriminate signals (difference limen, or DL) and how perceivers match signals (point of subjective equality, or PSE) are measured by the method of constant stimuli. In this method, perceivers are presented with a fixed set of stimuli (Engen, 1971). From the perceiver's responses to this set of stimuli, the perceiver's psychometric function can be estimated: The slope of the function indicates the discriminability of the signals, and the intercept of the function indicates the stimulus level that matches a specified criterion (such as perceiver is aware of signal $75 \%$ of the time or perceiver judges signal to be greater than another signal $50 \%$ of the time).

Newer psychophysical methods adapt stimulus levels to an individual perceiver's sensory ability. These adaptive methods are more efficient because earlier trials guide the selection of stimuli presented later; stimuli distant from the perceiver's threshold yield little information and are not presented. Some adaptive methods select stimulus levels by applying a maximum likelihood approach. In a maximum likelihood approach, the procedure posits

I thank Steve Keele for his guidance and support, and Richard Ivry for discussion and use of this procedure in some studies. I also thank Kathy Hawk, who ran many of the program simulations and SPSS analyses. 1 also thank Eric Gold, Asher Cohen, Yoav Cohen, Rick Wildes, Jacob Beck, Joshua Hurwitz, Scott Chaiken, and Ellen Hall for commentary on this procedure or on the manuscript. I especially thank an anonymous reviewer, who pointed out that this procedure would be appropriate for investigating Müller-Lyer-like illusions in which perceivers exhibit a constant error. Requests for reprints should be sent to R.A. Pokorny, AFRL/HEAB, 7909 Lindbergh Dr., Brooks AFB, TX 78235-5253 (e-mail: pokorny@alhrm.brooks.af.mil). a number of different possible models, each with a different psychometric function. Then, the observed data are compared with each model. The model that most closely matches the data (the most likely model) identifies the best current estimate of the perceiver's threshold. On the next trial, the procedure uses the best current estimate of the threshold to select the most informative stimulus step. (For an excellent recent review of adaptive psychophysical methods, see Treutwein, 1995.)

Two methods that describe a maximum likelihood approach to estimating perceptual thresholds are Pentland's Best PEST (Lieberman \& Pentland, 1982), and Watson and Pelli's QUEST (Watson \& Pelli, 1983). (Green, 1991, describes another maximum likelihood approach used to find perceptual thresholds but does not provide program listings; Shelton, 1983, made program listings available by writing to the author.) Best PEST differs from QUEST in two ways. First, Best PEST assumes a logistic distribution as the family of models for which maximum likelihood fits are sought, whereas QUEST assumes a Weibull distribution. Although the two underlying distributions have generally similar shapes, the logistic distribution approximates a normal distribution and is easier to compute than the normal distribution. Second, Best PEST, as described in Lieberman and Pentland (1982), finds only one threshold along the psychometric function, whereas QUEST finds any threshold along the psychometric function.

The current paper extends Best PEST to find thresholds anywhere along a perceiver's psychometric function. With such an extension, maximum likelihood estimation can be used to find any threshold along dimensions that follow a logistic distribution. Before describing how Best PEST can be modified, we will first consider experimental situations for which such a procedure would be helpful.

One perceptual investigation for which the modified Best PEST is well suited studies the comparative loudness 
of different wavelength sounds. For example, how does the loudness of a 440- $\mathrm{Hz}$ trumpet sound compare with that of a 256- Hz human voice sound? We might naturally want to use the simple Best PEST to find the PSE. But the simple Best PEST finds only one threshold, the PSE. This PSE estimate may be contaminated by response bias. By using the modified Best PEST to find thresholds at which the perceiver judges one signal to be louder and softer than another, we can check on response biases and get a clearer picture of the PSE, as well as an estimate of the DL.

Another common alternative to the Best PEST task is a two-alternative forced-choice (2AFC) task. Typically, in using an adaptive 2AFC task, a standard stimulus is presented in either the first or the second alternative (position or interval). The other signal, which we will specify as standard plus delta, is presented in the other alternative. When the perceiver correctly judges in which alternative the standard plus delta stimulus was presented, delta is decreased, so that the standard signal and the standard plus delta signal will be more similar on the next trial. When the perceiver judges incorrectly, delta is increased, and the standard signal and the standard plus delta signal are more different.

If an experimenter considers using a $2 \mathrm{AFC}$ task to study how a $440-\mathrm{Hz}$ signal compares in loudness to a $440-\mathrm{Hz}$ signal, the experimenter can assume that both signals are equally loud when presented at equal intensities. But when comparing a $256-\mathrm{Hz}$ signal to a $440-\mathrm{Hz}$ signal, the experimenter will not know the intensity of a $256-\mathrm{Hz}$ signal that equals a $440-\mathrm{Hz}$ signal. Thus, the $2 \mathrm{AFC}$ task does not apply, because the assumption that the standard of the two signals will be the same cannot be met. By using the modified Best PEST, we will be able to study directly the sensory equivalence and the comparison judgments of signals of different wavelengths. (To see simulations comparing a $2 \mathrm{AFC}$ task and the modified Best PEST in these situations, see Experiment 9.)

A second psychological question for which the modified Best PEST is appropriate is the investigation of constant error illusions. With the Müller-Lyer illusion, for example, two lines of identical length are perceived to be of different lengths because of the context of their presentation. The modified Best PEST procedure allows investigators to measure the slope of the psychophysical function of perceived line lengths as well as the absolute magnitude of the illusion. With the Müller-Lyer illusion, investigators could use the modified Best PEST to address questions such as (1) do perceivers who see the line length more accurately (i.e., those who are less affected by the illusion) also compare line lengths more accurately (i.e., have a steeper psychophysical function), and (2) are the slopes of the psychophysical function the same on either side of the PSE?

A third psychological question for which the modified Best PEST is well suited investigates how the memory trace of a signal is affected by a simultaneous (dual) task. In a dual task experiment in which one task is perception, the perceiver is presented with a standard signal, performs an intervening task, and then judges the comparison signal. By using the modified Best PEST, the effect of the intervening task on the PSE and the DL of the standard signal can be measured.

The rest of this paper (1) describes how to modify Best PEST to find any threshold along the psychometric function and (2) reports simulations that show how accurately and reliably Best PEST estimates thresholds. In explaining the modification, the paper describes a procedure for estimating one threshold anywhere along the psychometric function. In reporting the simulations, however, the paper reports how accurately and reliably the modified Best PEST estimates two thresholds. This paper explains how the procedure is modified to find a single threshold anywhere along the psychometric function, so the critical changes are highlighted. The experimenter or progammer can then modify this procedure for any experimental situation required. The simulations report finding two thresholds because the modified Best PEST will typically be used to find two thresholds. Finding two thresholds allows experimenters to estimate both the PSE and the DL, whereas finding only one threshold allows possible response bias to cloud the interpretation. Thus, the simulations reported in this paper search for two thresholds: one above the PSE and the other below the PSE, with both equally distant from the PSE. The trials for finding the higher threshold and the lower threshold should be randomly intermixed, so that the subject has a $50 \%$ chance of responding greater than or less than on every trial. With this procedure, the modified Best PEST can estimate the PSE by averaging the two thresholds and can estimate the DL from the difference between the two thresholds.

\section{MODIFICATION OF BEST PEST}

Best PEST and other maximum likelihood approaches (Green, 1991; Harvey, 1986, Watson \& Pelli, 1983) all assume an underlying form of the psychometric function and estimate parameters that yield a best fit to the observed data. Best PEST assumes a logistic distribution that has two parameters. Best PEST fixes the slope parameter and estimates the intercept parameter. The intercept specifies the stimulus value at which the perceiver responds greater than (or Yes, or some other criterion) as a specified percentage of the time (e.g., judgments of "greater than" $75 \%$ of the time). Then Best PEST computes the likelihood that each of the possible stimulus levels provides the best fit to the data. The stimulus level that provides the best fit is the estimate of the intercept and identifies the threshold. (For a fuller explanation of maximum likelihood approaches, see Harvey, 1986; MacMillan \& Creelman, 1991.)

To use Best PEST, the experimenter must first determine the range of possible thresholds that any perceiver might exhibit. Best PEST must present any stimulus value across the range of possible threshold values. The range of possible stimuli is then divided into a set of discrete 
steps. Associated with each stimulus step is a probability that reflects the likelihood that the corresponding stimulus step is the threshold sought. Thus, this array of probability values is called the threshold probability array. At any time in a series of trials, the stimulus step with the highest threshold probability value is the current best estimate of the threshold (see King-Smith, Grigsby, Vingris, Benes, \& Supowit, 1994, for different ways of estimating the threshold from a threshold probability array).

Best PEST assumes a logistic function, which generally has the form, $p(+)=[1+\exp -L]^{* *}-1$, where $L$ refers to the stimulus level. In Best PEST, $L$ is replaced with $(x-m) / s$, where $x$ is the stimulus level and ranges across all possible stimulus levels, $m$ is the intercept (the estimated threshold), and $s$ is the slope. After every trial, Best PEST updates all of the threshold probability values by multiplying the existing values by a logistic ogive. If the perceiver responds greater than, the threshold probability values are changed so that lower stimulus steps have higher values (i.e., the estimated threshold is lowered). If the perceiver responds less than, threshold probability values are changed so that higher stimulus steps have relatively higher values. Best PEST implements this procedure in the following PASCAL statement:

for $x:=$ minstim to maxstim

$$
\begin{aligned}
\text { threshold-prob }[x]:= & \text { threshold-prob }[x] \\
& *\left(1 /\left(1+e^{* *}-r((m-x) / s)\right)\right) .
\end{aligned}
$$

(This implementation is for a $\mathrm{Y} / \mathrm{N}$ task, not a $2 \mathrm{AFC}$ task. In a $\mathrm{Y} / \mathrm{N}$ task, the perceiver's response rate of saying that one signal is greater than another signal ranges from 0 to 1 ; in a $2 \mathrm{AFC}$, the response rate ranges from .5 to 1 .)

The adjusting logistic ogive depends on three factors: (1) its direction, (2) its slope, and (3) its position relative to the threshold probability values. Each of these will be discussed in turn.

The direction-whether the adjusting logistic ogive slants up or down-depends on the perceiver's response. If the perceiver responds greater than, the stimulus presented was too large, and the estimated threshold should decrease. The adjusting logistic ogive assigns high values to low-stimulus steps and slopes down from low-stimulus steps to high-stimulus steps. If the perceiver responds less than, the stimulus was too small, and the stimulus level of the estimated threshold should increase. The slope of the adjusting logistic ogive will now slope up from lowstimulus values to high.

The parameter controlling the direction of the adjusting ogive is $r$, the perceiver's response, which is -1 for less than or +1 for greater than.

The slope of the adjusting logistic ogive refers to the steepness of the adjusting ogive. This slope is defined by the change in response rate (the $y$-axis of the psychometric function) over the change in stimulus levels (the $x$ axis). The response rate with a logistic distribution can be defined in logits, which corresponds to $L$ of the basic logistic formula: When $L$ is 0 , the probability of responding greater than is $.5[p(+)$ is .5$]$; when $L$ is $1, p(+)$ is .73 ; when $L$ is $-1, p(+)$ is .27. Each unit change of $L$ (i.e., from 0 to 1 , or from -3 to -2 ) is called a logit. The change in stimulus steps refers to the number of stimulus steps it takes to go from 1 logit to another. A logistic ogive that uses only 5 steps to traverse 1 logit has twice the slope of an ogive that uses 10 steps. It might seem that, to estimate the perceiver's threshold correctly, the slope of the adjusting ogive assumed by Best PEST must approximate the slope of the perceiver's psychometric function. Earlier studies, however, found that Best PEST accurately estimates a perceiver's PSE despite large differences between the slope of the perceiver's psychometric function and that assumed by Best PEST (Pentland, 1980). The possibility that the slope of the adjusting ogive must approximate the perceiver's psychometric function to accurately estimate thresholds will be carefully scrutinized when the modified Best PEST is tested.

The parameter indicating the slope of the adjusting ogive in the implementation is $s$. If $s$ is 5, the slope of the adjusting ogive is 1 logit for every 5 stimulus levels. If $s$ is 12 , the adjusting ogive is less steep, with 1 logit for every 12 stimulus levels.

The position of the adjusting logistic ogive, relative to the stimuli levels, anchors one point of the adjusting logistic ogive to one stimulus step. Then steps along the threshold probability array can be multiplied by specific values of the logistic ogive.

The parameters controlling the position of the adjusting ogive in the implementation are $(m-x)$. The value of $(m-x)$ positions the adjusting ogive, relative to the threshold probability array. The stimulus step just presented for measurement is indicated by $m$. The range of $x$ is from the minimum stimulus step to the maximum stimulus step. To position the adjusting ogive relative to the set of stimuli steps, the adjusting ogive and the stimulus steps are tied together by the estimated threshold. At the estimated threshold, the adjusting ogive has the value of .5 (as the procedure searches for the PSE, where $p(+)=.5$ ). At the estimated threshold, $L$ (from the logistic formula) has the value of 0 . For $L$ to be 0 in this implementation, $(m-x)$ is 0 ; if $(m-x)$ is $0, m$ must equal $x$. Putting together the value of the logistic formula at the estimated threshold (.5) with the value of $L$ at the estimated threshold $(0)$, the adjusting ogive, which substitutes " $r(m-x) / s$ " for $L$, has a value of .5 at stimulus step $m$.

To estimate thresholds other than the PSE, Best PEST can be modified to find a threshold anywhere along the perceiver's psychometric function. The threshold sought can be identified by specifying $L$ from the logistic formula: When $L$ is $0\left[(1+\exp 0)^{* *}-1\right]$, the threshold specified is the PSE (where the perceiver responds greater than and less than $50 \%$ of the time). When $L$ is 1 , the threshold specified is 1 logit above the PSE, the stimulus level where the perceiver responds greater than $73 \%$ of the time. When $L$ is -1 , the threshold specified is 1 logit below the PSE, where the perceiver responds greater than $27 \%$ of 
Table 1

Estimating PSEs and DLs When Perceivers' Slope Differs From That Assumed by Best PEST

\begin{tabular}{|c|c|c|c|c|c|c|c|c|c|}
\hline \multirow{2}{*}{\multicolumn{2}{|c|}{$\begin{array}{l}\text { Simulated } \\
\text { Perceivers } \\
\end{array}$}} & \multicolumn{4}{|c|}{$\begin{array}{l}\text { Estimated Thresholds } \\
\text { From Best PEST }\end{array}$} & \multicolumn{4}{|c|}{$\begin{array}{l}\text { Estimated Thresholds } \\
\text { From PEST and Regression }\end{array}$} \\
\hline & & \multicolumn{2}{|c|}{ PSE } & \multicolumn{2}{|c|}{$\mathrm{DL}$} & \multicolumn{2}{|c|}{ PSE } & \multicolumn{2}{|c|}{$\mathrm{DL}$} \\
\hline PSE & $\mathrm{DL}$ & $M$ & $S D$ & $M$ & $S D$ & $M$ & $\widehat{S D}$ & $M$ & $\overrightarrow{S D}$ \\
\hline 0 & 3 & -0.02 & 1.25 & 3.31 & 1.31 & -0.01 & 0.92 & 2.86 & 0.70 \\
\hline 0 & 6 & 0.04 & 1.88 & 6.46 & 1.98 & 0.02 & 1.70 & 5.71 & 1.48 \\
\hline 0 & 9 & -0.03 & 2.60 & 9.46 & 2.56 & -0.02 & 2.58 & 8.34 & 2.21 \\
\hline 0 & 12 & 0.31 & 3.28 & 12.38 & 3.20 & 0.32 & 3.35 & 11.12 & 2.99 \\
\hline 0 & 18 & 0.22 & 4.89 & 17.50 & 4.90 & 0.14 & 5.06 & 16.83 & 6.61 \\
\hline
\end{tabular}

the time. When $L$ is 2 , the threshold specified is 2 logits above the PSE, where the perceiver responds greater than $88 \%$ of the time.

To modify the Best PEST implementation, a new term must be added so that $L$, the value of the exponent from the original logistic formula, is reflected in the implementation. (In the unmodified Best PEST, $L$ need not be stated, as the implementation finds the threshold where $L$ is 0 .) To incorporate an $L$-score term, remember that, when searching for the threshold where $L$ is $0, L$ is implemented with " $r(m-x) / s$ ". In the new implementation, the exponent, which should yield a value of $L$ when $m$ equals $x$ is " $r\left(m-x+L\right.$ score $\left.^{*} s\right) / s$ ". This implementation will first be explained and then tested.

One way to understand how the modified Best PEST searches for any threshold is that we are using the standard maximum likelihood procedure, with a different basic function to be sought. Instead of searching for the intercept of the logistic distribution when $p(+)=[1+\exp -(x-m)$ $/ s] * *-1$, we are searching for a different function: $p(+)=\left[1+\exp -\left(x-m+L\right.\right.$ score $\left.\left.\left.^{*} s\right) / s\right)\right]^{* *}-1$. We are still finding $m$, the intercept, but with a different function.

\section{PERFORMANCE OF THE MODIFIED BEST PEST}

To test how well the modified Best PEST works, Monte Carlo experiments examined how accurately the modified Best PEST estimated perceivers' thresholds across many different conditions.

In these experiments, the Best PEST procedure selects stimuli and estimates thresholds as it would when conducting experiments on real perceivers. The modified Best PEST procedure used 97 stimulus steps, numbered from -48 to +48 . The assumed PSE was at stimulus step 0 ; the assumed slope was 12 stimulus steps per logit. Usually two thresholds were estimated per session. Sessions consisted of 72 trials (this was varied in Experiment 3), with 36 trials used to find thresholds 1 logit above and below the PSE (this was varied in Experiment 4). The PSE is computed from the average of the two thresholds, and the DL is computed by taking the difference between the two thresholds and dividing by two. The thresholds reported are based on at least 400 estimates of each threshold.
The responses of real perceivers are replaced with a computerized response generator that has a logistic psychometric function. Responses are either greater than or less than, depending on the stimulus level presented and the simulated perceiver's PSE and DL. For example, when simulating a perceiver whose PSE is 5 and DL is 15 , if PEST presents the perceiver stimulus step 5 , a random number generator creates greater than responses half of the time; if the PEST presents stimulus step 20 (for this perceiver, 1 logit above the PSE), the simulator responds greater than on $73 \%$ of the trials (corresponding to the response rate 1 logit above the PSE); if the perceiver is presented stimulus step -10 ( 1 logit below the assumed PSE), the simulated perceiver responds greater than $27 \%$ of the time.

The following experiments check how well the modified Best PEST estimates perceivers' thresholds when the characteristics of the modified Best PEST and the perceiver are known and systematically varied. For each threshold found, two estimates are reported. The first estimate is the threshold reported directly by the modified Best PEST: After PEST has run its course of trials, the stimulus step with the highest threshold probability is PEST's best estimate of the threshold. A second threshold estimate from each PEST session is generated by performing a logistic regression on all of the trials presented during the Best PEST procedure. The data were regressed for each session by SPSS, which uses a maximum likelihood approach to estimate the intercept parameter.

\section{Estimation of a Sensory Threshold \\ Besides the Perceiver's PSE}

This experiment addresses whether the modified Best PEST accurately estimates the perceiver's thresholds (PSE and DL) even when the perceiver's slope differs from that assumed by the PEST procedure. To address this issue, the modified Best PEST estimated thresholds for perceivers whose psychometric functions had slopes ranging from 1 logit per 3 stimulus steps to 1 logit per 18 stimulus steps. The modified Best PEST procedure assumed a psychometric function of 1 logit per 12 steps. Table 1 shows how accurately the modified Best PEST measured perceivers' PSEs and DLs.

The threshold estimates of the perceiver's DL from the modified Best PEST procedure closely followed the true 
Table 2

Estimating a Threshold When Perceivers' PSE is Not at the Expected Stimulus Step

\begin{tabular}{|c|c|c|c|c|c|c|c|c|c|}
\hline \multirow{2}{*}{\multicolumn{2}{|c|}{$\begin{array}{l}\text { Simulated } \\
\text { Perceivers } \\
\end{array}$}} & \multicolumn{4}{|c|}{$\begin{array}{l}\text { Estimated Thresholds } \\
\text { From Best PEST }\end{array}$} & \multicolumn{4}{|c|}{$\begin{array}{l}\text { Estimated Thresholds } \\
\text { From PEST and Regression }\end{array}$} \\
\hline & & \multicolumn{2}{|c|}{ PSE } & \multicolumn{2}{|c|}{ DL } & \multicolumn{2}{|c|}{ PSE } & \multicolumn{2}{|c|}{ DL } \\
\hline PSE & DL & $M$ & $S D$ & $M$ & $S D$ & $M$ & $S D$ & $M$ & $S D$ \\
\hline \multirow[t]{3}{*}{12} & 6 & 12.01 & 2.00 & 6.3 & 1.9 & 12.07 & 1. & 5.42 & 1.45 \\
\hline & 12 & 11. & 3.2 & 12. & 3.1 & 11. & 3. & 11.60 & 3.42 \\
\hline & 18 & 11.33 & 4.63 & 17.67 & 4.75 & 11.97 & 4.76 & 16.71 & 4.84 \\
\hline \multirow[t]{3}{*}{-24} & 6 & -24.14 & 1.94 & 6.48 & 1.92 & -24.04 & 1.72 & 5.67 & 1.43 \\
\hline & 12 & -23.54 & 3.19 & 12.09 & 3.21 & -23.77 & 3.32 & 10.95 & 3.12 \\
\hline & 18 & -22.52 & 4.44 & 17.05 & 4.52 & -23.91 & 4.76 & 16.79 & 5.08 \\
\hline
\end{tabular}

DL of the simulated perceiver; the slope of the psychometric function assumed by the modified Best PEST procedure (12 steps/logit) had virtually no effect on the estimated threshold.

The threshold estimates produced directly from Best PEST are similar to the threshold estimates produced by submitting the trials to logistic regression (for perceivers with identical slopes, the estimated thresholds correlate about .9). The thresholds estimated by submitting the trials selected by Best PEST to a logistic regression generally yielded more reliable estimates of the threshold (smaller standard deviations of the estimate) than did the threshold estimates produced directly by the modified Best PEST. So, when a logistic regression system is available, the threshold estimated by submitting experimental trials from the Best PEST procedure to logistic regression is superior to the threshold estimated directly from the Best PEST procedure.

\section{Estimation of Sensory Thresholds \\ When the Perceiver's PSE Differs \\ From That Assumed by Best PEST}

Experiment 1 showed that modified Best PEST accurately estimates perceivers' DLs, regardless of whether the perceivers' psychometric slope equaled that assumed by the procedure. In Experiment 1, although perceivers' perceptual slope differed from that assumed by the modified Best PEST procedure, the perceivers' PSE always equaled that assumed by the procedure. The next experiment examined whether the modified Best PEST accurately estimates thresholds, even when the perceivers' PSE differs from that assumed by the procedure.

As is indicated in Table 2, the modified Best PEST accurately estimates perceivers' thresholds, even when perceivers' PSEs differ from that assumed by the procedure.

\section{Estimation of Sensory Thresholds When the Number of Trials per Estimate Varies}

How well does the modified Best PEST estimate thresholds when different numbers of trials are used? Might biases appear with fewer trials per estimate? Is there an optimally efficient number of trials for estimating a threshold?

To answer these questions, the modified Best PEST estimated thresholds while varying the number of trials per estimate from 18 to 288 trials. To identify the most efficient number of trials per estimate, the variance of the sampling distribution (given that 288 trials are divided into different-sized blocks) is shown in the last column of Table 3.

As the number of trials per estimate increases, the estimate becomes more accurate, and the reliability im-

Table 3

How Varying the Number of Trials Affects the Estimated Threshold

\begin{tabular}{|c|c|c|c|c|c|c|c|c|c|c|}
\hline \multirow{3}{*}{$\begin{array}{l}\text { Number } \\
\text { of Trials }\end{array}$} & \multicolumn{4}{|c|}{$\begin{array}{l}\text { Estimated Thresholds } \\
\text { From Best PEST } \\
\end{array}$} & \multicolumn{4}{|c|}{$\begin{array}{c}\text { Estimated Thresholds } \\
\text { From PEST } \\
\text { and Regression } \\
\end{array}$} & \multicolumn{2}{|c|}{$\begin{array}{c}\text { Standard Deviation } \\
\text { of DL/No. of } \\
\text { Blocks of Trials } \\
\text { Given } 288 \text { Trials } \\
\end{array}$} \\
\hline & \multicolumn{2}{|c|}{ PSE } & \multicolumn{2}{|c|}{ DL } & \multicolumn{2}{|c|}{ PSE } & \multicolumn{2}{|c|}{ DL } & \multirow{2}{*}{$\begin{array}{l}\text { Best } \\
\text { PEST }\end{array}$} & \multirow{2}{*}{$\begin{array}{r}\text { PEST } \\
\text { and Reg } \\
\end{array}$} \\
\hline & $M$ & $S D$ & $M$ & $S D$ & $M$ & $S D$ & $M$ & $S D$ & & \\
\hline 18 & -0.18 & 4.28 & 8.07 & 3.98 & -0.10 & 4.27 & 5.41 & 2.61 & 1.0 & .65 \\
\hline 36 & 0.14 & 2.62 & 6.77 & 2.80 & 0.11 & 2.37 & 5.38 & 1.82 & .99 & .64 \\
\hline 72 & 0.03 & 1.95 & 6.30 & 1.97 & 0.05 & 1.78 & 5.36 & 1.36 & .99 & .68 \\
\hline 144 & 0.02 & 1.35 & 6.18 & 1.38 & 0.00 & 1.12 & 5.30 & 1.07 & .98 & .76 \\
\hline 288 & -0.02 & 0.98 & 6.05 & 0.97 & 0.00 & 0.85 & 5.85 & 0.78 & .97 & .78 \\
\hline
\end{tabular}

Note-Simulation perceiver has PSE of 0. DL of 6 . In the last column, standard deviation of DL/number of blocks of trials given 288 trials is computed by taking the variance of the DL and dividing by the number of blocks of trials for each block size $(18,36,72,144$, or 288), given 288 trials in total (i.e., $16,8,4,2$, and 1). For example, with 18 trials per block, the Best PEST estimate of DL had a variance of 3.98 squared, or 15.88. 15.88 divided by 16 (since there could be 16 estimates of the DL with 288 trials, and 18 trials per estimate), yields a variance of .9925 . The square root of this number yields the standard deviation, .996 , or, rounded to two digits, 1.0 . 
Table 4

Estimating Various Thresholds Along the Psychometric Function

\begin{tabular}{|c|c|c|c|c|c|c|c|c|c|c|}
\hline \multirow{2}{*}{\multicolumn{2}{|c|}{$\begin{array}{l}\text { Simulated } \\
\text { Perceiver }\end{array}$}} & \multirow{3}{*}{$\begin{array}{c}\text { Threshold } \\
\text { Sought } \\
\frac{L}{}\end{array}$} & \multicolumn{4}{|c|}{$\begin{array}{l}\text { Estimated Threshold } \\
\text { from Best PEST }\end{array}$} & \multicolumn{4}{|c|}{$\begin{array}{l}\text { Estimated Threshold From } \\
\text { Best PEST and Regression }\end{array}$} \\
\hline & & & \multicolumn{2}{|c|}{ PSE } & \multicolumn{2}{|c|}{$\mathrm{DL}$} & \multicolumn{2}{|c|}{ PSE } & \multicolumn{2}{|c|}{$\mathrm{DL}$} \\
\hline PSE & $\mathrm{DL}$ & & $M$ & $S D$ & $M$ & $S D$ & $M$ & $S D$ & $M$ & $S D$ \\
\hline \multirow[t]{8}{*}{0} & 6 & \pm .5 & -0.27 & 2.39 & 6.35 & 3.63 & -0.09 & 1.56 & 5.25 & 1.80 \\
\hline & 6 & \pm 1.0 & -0.02 & 2.02 & 6.26 & 1.89 & -0.01 & 1.80 & 5.59 & 1.34 \\
\hline & 6 & \pm 1.5 & 0.09 & 1.85 & 6.45 & 1.37 & 0.06 & 2.03 & 5.75 & 1.14 \\
\hline & 6 & \pm 2.0 & 0.02 & 1.86 & 6.71 & 1.46 & -0.01 & 2.43 & 5.97 & 1.26 \\
\hline & 12 & \pm .5 & 0.19 & 4.27 & 11.87 & 6.04 & 0.16 & 3.06 & 10.02 & 4.15 \\
\hline & 12 & \pm 1.0 & -0.23 & 3.35 & 12.29 & 3.34 & -0.26 & 3.46 & 11.20 & 3.43 \\
\hline & 12 & \pm 1.5 & -0.09 & 2.99 & 12.27 & 2.60 & -0.14 & 3.66 & 11.51 & 2.64 \\
\hline & 12 & \pm 2.0 & -0.39 & 3.27 & 12.36 & 2.16 & -0.41 & 4.62 & 11.68 & 2.28 \\
\hline
\end{tabular}

Note-the DL estimated from the Best PEST has been scaled by the threshold sought. For example, Best PEST found a threshold of about 24.7 stimulus steps with a standard deviation (SD) of 4.3 when searching for the threshold of \pm 2 . But since we want to estimate the threshold one DL away from the PSE, the measured threshold is divided by 2 , as is the $S D$, to yield a DL of 12.36 and an $S D$ of about 2.15 .

proves. But varying the number of trials per estimate does not appreciably affect the efficiency of threshold estimate reliability. For the estimated threshold directly from the Best PEST procedure, the reliability is approximately equal, regardless of using more trials per block with fewer blocks or fewer trials per block with more blocks. For the estimated threshold from the Best PEST and logistic regression, using from 36 to 72 trials per block with more blocks may lead to slightly greater reliability.

\section{Estimation of Sensory Thresholds \\ at Different Points Along the Perceiver's Psychometric Function}

Experiment 3 showed that the reliability of the thresholds estimated by the modified Best PEST depends on the total number of trials run; dividing the trials into blocks of different sizes has little effect on reliability. The next study explored how efficiently the modified Best PEST estimates thresholds at points other than those 1 logit away from the perceiver's PSE. That is, will finding thresholds 1.5 logits away from PSEs be more efficient than finding thresholds 1 logit away from the PSE? In this study, the thresholds sought are $0.5,1,1.5$, and 2 logits away from the PSE.

Table 4 shows that the most reliable estimates of perceivers' thresholds are obtained when searching for thresholds which are 1.5 logits and farther away from the PSE. Searching for the thresholds 0.5 logits away from the PSE leads to far worse reliability than when searching for thresholds farther away from the PSE. For perceivers with steeper psychometric functions than that assumed by the PEST procedure, the reliability appears to be better when searching for the threshold 1.5 logits away, rather than 1 logit away, from the PSE: For a perceiver with a DL of 6, searching for the threshold 1.5 logits away from the PSE, rather than 1.0 logits away from the PSE, decreases the standard deviation from 1.89 to 1.37 .

The practical experimenter, in addition to considering which threshold sought leads to the most reliable estimates of the threshold, should also consider the ease with which subjects make judgments. On one hand, perceivers are more comfortable judging stimuli further from the PSE, as some trials are relatively easy. On the other hand, if many trials are far from the perceiver's PSE, the perceiver's attention may wander, and he or she may perform suboptimally. The effect of lack of attention, or lapses, is addressed next.

\section{Estimation of Sensory Thresholds When Perceiver's Attention Lapses}

Maximum likelihood procedures have been criticized for being unforgiving when perceivers make errors or fail to pay attention (Emerson, 1984; Taylor, Forbes, \& Creelman, 1983). This section explores the effect of the perceiver's failing to pay attention to the stimulus. The perceiver's inattention is simulated by responding randomly, independent of the stimulus (half greater than, half less than responses) on $20 \%$ of the trials. Results are shown in Table 5.

When searching for the PSE, the estimated threshold is not biased when the perceiver's attention lapses, although reliability is lower. (As a side note, this table shows that, when the threshold sought is the PSE, using logistic regression to estimate the DL is inaccurate and unreliable.) But when searching for thresholds other than the PSE, the estimated threshold is biased toward more extreme sensory values (away from the PSE).

This bias may be understood in the following way. When searching for the threshold at which $p(+)=.73$, less than responses raise the estimated threshold more than greater than responses lower it. On those trials on which the perceiver fails to pay attention, he or she is assumed to respond greater than and less than equally. Since less than responses have a greater effect on the threshold estimate than do greater than responses, the estimated threshold is biased in the direction of less than responses; the estimated threshold will be higher than the perceiver's true $p(+)=.73$ threshold.

Can lapses be differentiated from perceptual acuity? Finney (1971) proposed that chi-square be used to deter- 
Table 5

Estimated Thresholds When Perceivers' Attention Lapses

\begin{tabular}{|c|c|c|c|c|c|c|c|c|c|c|c|}
\hline \multirow{2}{*}{$\begin{array}{l}\text { Simulated } \\
\text { Perceivers }\end{array}$} & \multirow{3}{*}{$\begin{array}{c}\text { Threshold } \\
\text { DL }\end{array}$} & \multirow[b]{3}{*}{ Sought } & \multirow[b]{3}{*}{$\%$ Lapses } & \multicolumn{4}{|c|}{$\begin{array}{l}\text { Estimated Thresholds } \\
\text { From Best PEST }\end{array}$} & \multicolumn{4}{|c|}{$\begin{array}{c}\text { Estimated Thresholds From } \\
\text { PEST and Regression }\end{array}$} \\
\hline & & & & \multicolumn{2}{|c|}{ PSE } & \multicolumn{2}{|c|}{ DL } & \multicolumn{2}{|c|}{ PSE } & \multicolumn{2}{|c|}{$\mathrm{DL}$} \\
\hline PSE & & & & $M$ & $S D$ & $M$ & $S D$ & $M$ & $S D$ & $M$ & $S D$ \\
\hline \multirow[t]{2}{*}{0} & 6 & 0 & 0 & 0.09 & 2.45 & & & 0.11 & 2.38 & 4.10 & 2.68 \\
\hline & & & 20 & 0.46 & 2.89 & & & 0.07 & 12.71 & 7.16 & 12.98 \\
\hline \multirow[t]{2}{*}{0} & 12 & 0 & 0 & 0.15 & 4.26 & & & 0.26 & 6.41 & 6.59 & 6.96 \\
\hline & & & 20 & 0.15 & 5.52 & & & 0.86 & 12.98 & 8.42 & 14.16 \\
\hline \multirow[t]{2}{*}{0} & 6 & \pm 1 & 0 & -0.13 & 1.93 & 6.45 & 1.80 & -0.11 & 1.73 & 5.62 & 1.33 \\
\hline & & & 20 & -0.13 & 3.71 & 9.81 & 3.58 & -0.12 & 3.60 & 8.75 & 3.10 \\
\hline \multirow[t]{2}{*}{0} & 12 & \pm 1 & 0 & 0.08 & 3.37 & 12.44 & 3.19 & 0.04 & 3.41 & 11.15 & 2.96 \\
\hline & & & 20 & 0.38 & 5.05 & 16.79 & 5.47 & 0.51 & 4.99 & 15.77 & 5.82 \\
\hline
\end{tabular}

Note-When searching for the threshold of 0 , the PSE, Best PEST could not estimate the DL; hence those cells are empty. Also note that 36 trials were used to find each threshold: When searching for the 0 threshold, 36 trials were used; when searching for +1 and $-1,72$ trials were used, 36 for each threshold.

mine whether variability can be attributable solely to sampling error or whether variability can be attributable to poor perceptual skill. Another approach is to look at how the Best PEST procedure estimates the probability values for stimulus values near the estimated threshold. This approach is addressed next.

\section{Estimation of Sensory Thresholds and Reliability of Threshold Estimates by Using Confidence Intervals Around the Estimated Absolute Threshold}

That lapses cause biases raises the question of the importance of detecting lapses or minimizing the effect of lapses. One avenue that has been suggested to mitigate the effect of lapses is to continue testing until the reliability of the estimated threshold exceeds a specified criterion. This reliability is measured by constructing confidence intervals around the estimated threshold by using the threshold probability array. One can sum the values in the threshold probability array within $n$ steps of the estimated threshold and divide that sum by the sum of probabilities from all possible stimulus steps. These confidence intervals have been used to determine when to stop searching for the threshold (Watson \& Pelli, 1983) and to show the reliability of estimated thresholds (Pentland, 1980). The width of the confidence intervals around the

Table 6

Confidence Intervals

\begin{tabular}{|c|c|c|c|c|c|c|c|c|}
\hline \multirow[b]{2}{*}{ No. of Trials } & \multirow{2}{*}{$\begin{array}{l}\text { Slope Assumed } \\
\text { by Best PEST }\end{array}$} & \multicolumn{2}{|c|}{$\begin{array}{l}\text { Simulated } \\
\text { Perceivers }\end{array}$} & \multirow[b]{2}{*}{$\%$ Lapses } & \multicolumn{2}{|c|}{$\begin{array}{c}\begin{array}{c}\text { Estimated Threshold } \\
\text { From Best Pest }\end{array} \\
\text { PSE }\end{array}$} & \multicolumn{2}{|c|}{$\begin{array}{l}\text { Confidence } \\
\text { Intervals } \\
(2 \text { Logits } \\
\text { Around PSE) }\end{array}$} \\
\hline & & PSE & $\mathrm{DL}$ & & $M$ & $S D$ & $M$ & $S D$ \\
\hline 18 & 6 & 0 & 6 & 0 & -0.41 & 3.62 & 5.84 & 0.19 \\
\hline 18 & 6 & 0 & 6 & 20 & 0.35 & 5.05 & 5.97 & 0.37 \\
\hline 18 & 6 & 0 & 6 & 50 & 0.72 & 12.26 & 5.98 & 0.29 \\
\hline 18 & 6 & 0 & 12 & 0 & 0.28 & 6.39 & 5.94 & 0.31 \\
\hline 18 & 6 & 0 & 12 & 20 & -0.10 & 8.93 & 5.91 & 0.24 \\
\hline 18 & 6 & 0 & 12 & 50 & -0.67 & 15.30 & 5.91 & 0.24 \\
\hline 18 & 12 & 0 & 6 & 0 & 0.33 & 3.82 & 9.32 & 0.20 \\
\hline 18 & 12 & 0 & 6 & 20 & 0.05 & 4.45 & 9.39 & 0.28 \\
\hline 18 & 12 & 0 & 6 & 50 & -0.29 & 10.20 & 9.39 & 0.26 \\
\hline 18 & 12 & 0 & 12 & 0 & -0.64 & 5.27 & 9.38 & 0.22 \\
\hline 18 & 12 & 0 & 12 & 20 & -0.41 & 9.05 & 9.41 & 0.26 \\
\hline 18 & 12 & 0 & 12 & so & 0.03 & 13.75 & 9.43 & 0.33 \\
\hline 36 & 6 & 0 & 6 & 0 & 0.13 & 2.36 & 4.62 & 0.07 \\
\hline 36 & 6 & 0 & 6 & 20 & -0.01 & 3.23 & 4.65 & 0.09 \\
\hline 36 & 6 & 0 & 6 & 50 & -0.46 & 6.30 & 4.67 & 0.15 \\
\hline 36 & 6 & 0 & 12 & 0 & -0.41 & 4.46 & 4.62 & 0.10 \\
\hline 36 & 6 & 0 & 12 & 20 & 0.98 & 6.32 & 4.65 & 0.12 \\
\hline 36 & 6 & 0 & 12 & 50 & 2.18 & 9.25 & 4.63 & 0.10 \\
\hline 36 & 12 & 0 & 6 & 0 & 0.56 & 2.20 & 7.06 & 0.06 \\
\hline 36 & 12 & 0 & 6 & 20 & -0.36 & 2.76 & 7.08 & 0.12 \\
\hline 36 & 12 & 0 & 6 & 50 & 0.93 & 7.84 & 7.18 & 0.20 \\
\hline 36 & 12 & 0 & 12 & 0 & -0.09 & 4.41 & 7.11 & 0.10 \\
\hline 36 & 12 & 0 & 12 & 20 & -0.24 & 5.51 & 7.16 & 0.16 \\
\hline 36 & 12 & 0 & 12 & 50 & -1.77 & 9.02 & 7.15 & 0.18 \\
\hline
\end{tabular}


Table 7

Estimated Thresholds After 12 Implicit Extreme Stimulus Presentations

\begin{tabular}{|c|c|c|c|c|c|c|c|c|c|}
\hline \multirow{2}{*}{\multicolumn{2}{|c|}{$\begin{array}{l}\text { Simulated } \\
\text { Perceivers }\end{array}$}} & \multicolumn{4}{|c|}{$\begin{array}{c}\text { Estimated Thresholds } \\
\text { From Best PEST }\end{array}$} & \multicolumn{4}{|c|}{$\begin{array}{l}\text { Estimated Thresholds } \\
\text { From PEST and Regression }\end{array}$} \\
\hline & & \multicolumn{2}{|c|}{ PSE } & \multicolumn{2}{|c|}{$\mathrm{DL}$} & \multicolumn{2}{|c|}{ PSE } & \multicolumn{2}{|c|}{ DL } \\
\hline PSE & $\mathrm{DL}$ & $M$ & $S D$ & $M$ & $S D$ & $\bar{M}$ & $S D$ & $M$ & $S D$ \\
\hline 0 & 3 & 0.03 & 1.25 & 3.22 & 1.13 & 0.05 & 0.86 & 2.80 & 0.72 \\
\hline 0 & 6 & 0.17 & 1.85 & 6.36 & 1.89 & 0.16 & 1.72 & 5.58 & 1.48 \\
\hline 0 & 9 & -0.08 & 2.57 & 9.49 & 2.28 & -0.10 & 2.62 & 8.74 & 2.06 \\
\hline 0 & 12 & 0.28 & 3.12 & 12.21 & 3.18 & 0.29 & 3.37 & 11.26 & 3.53 \\
\hline 0 & 18 & 0.31 & 3.98 & 16.95 & 3.90 & 0.34 & 4.64 & 16.66 & 4.98 \\
\hline
\end{tabular}

estimated threshold in various conditions is shown in Table 6.

Unfortunately, the simulations shown in Table 6 indicate that these confidence intervals constructed from the threshold probability are not sensitive to the reliability of subjects' judgments. Simulated perceivers with $50 \%$ lapses have the same confidence intervals as perceivers with no lapses. The confidence intervals around the estimated threshold depend on the number of trials and the steepness of the psychometric function assumed by Best PEST; neither the perceiver's psychometric function nor the perceiver's attention (or lack thereof) has any affect on the confidence intervals.

Confidence intervals obtained from the threshold probability array should not be used to estimate the perceiver's sensory ability.

\section{Use of A Priori Information}

Prior information is used in threshold estimation procedures in three different ways. First, both adaptive methods and traditional fixed stimulus methods use prior information for determining the range of stimuli to present. Second, adaptive methods use prior information from earlier trials in an experimental session for selecting stimuli to be presented on later trials. Third, some adaptive procedures use knowledge about the parameters of the psychometric function to guide the selection of stimuli presented, this prior knowledge is helpful early in an experimental session, when little information has been obtained from earlier trials. This section discusses this third kind of information, foreknowledge of the perceiver's psychometric function. The discussion first will describe why foreknowledge is helpful; second, how foreknowledge is included in the modified Best PEST; and third, how foreknowledge affects accuracy and reliability of threshold estimates from the modified Best PEST.

To understand why foreknowledge of the psychometric function is helpful, consider the selection of stimuli presented if nothing is assumed about the shape of the perceiver's psychometric function. With no assumptions about the estimate of the thresholds, the threshold probabilities for all possible stimulus values are equal, and the PEST procedure selects the middle step. If the perceiver responds less than, the adjusting ogive changes the threshold probability array to slant up, and the next stimulus presented is the absolute maximum stimulus step presentable. The general problem is that, when prior information from earlier trials is not available (when there are no or few earlier trials) and foreknowledge about the likely shape of the perceiver's psychometric function is not used, extreme stimulus values are presented.

Various ways can be used to instill prior information into the threshold probability array. Best PEST uses implicit trials. Implicit trials assume that, when the minimum or maximum stimulus levels are presented, the perceiver will respond correctly. Pentland's Best PEST uses one cycle of implicit trials, including one minimum stimulus level, which the perceiver is assumed to respond less than to, and one maximum stimulus level, which the perceiver is assumed to respond greater than to. Watson and Pelli (1983) describe how to input an assumed psychometric function into the threshold probability array and how to analyze the data so that the assumed psychometric function does not affect the final maximum likelihood estimate of the threshold. Harvey (1986) discusses four methods of reducing presentation of extreme stimulus steps on early trials, including the two mentioned above. Madigan and Williams (1987) report simulations in which Best PEST is modified with a QUEST-like initialization of the threshold probability array; they report that including foreknowledge of the psychometric function improves the accuracy of the threshold estimates.

To simply initialize a prior distribution of threshold values into the threshold probability array, the modified Best PEST can be run with any number of implicit trials. Pentland's Best PEST uses one implicit maximum stimulus step and one implicit minimum stimulus step. To determine how many implicit minimum and maximum trials to present, a rule from the original PEST (Taylor \& Creelman, 1967) was applied: The largest stimulus change should be half of one standard deviation of the assumed psychometric function. So, if the procedure assumes 12 stimulus steps for 1 logit, the largest stimulus step change from one trial to the next should be 6 steps. In varying the number of implicit trials, presenting $12 \mathrm{implicit}$ trials led to 6 stimulus steps being the largest change. Table 7 shows the results of threshold estimates when $12 \mathrm{implicit}$ trials preceded the explicit presentation of stimuli to the simulated perceiver.

After implicitly presenting 12 cycles $(1$ cycle means implicit presentation of both the minimum and the maximum stimulus levels), the modified Best PEST generally 
estimates thresholds accurately and reliably. The simulations show that, when the perceiver's slope is steeper than that assumed by the Best PEST procedure, the accuracy and reliability are superior to or comparable with the threshold estimates when only 1 implicit trial cycle is used. When the perceiver's slope is shallower than that assumed by the procedure, the modified Best PEST thresholds tend to underestimate the threshold.

Installing prior information into the threshold probability array, besides reducing the presentation of extreme stimulus values to perceivers, may explain a disparity among reports describing adaptive procedures. Some reports of adaptive methods claim that Best PEST and similar adaptive procedures produce unbiased threshold estimates (Pentland, 1980), whereas other reports describe biased threshold estimates from adaptive procedures (Emerson, 1986; Simpson, 1989). Different results might reflect different uses of prior information to initialize the threshold probability array. If prior information is not used at all, the early stimulus presentations will include extreme stimulus values, from which the adaptive procedure may not completely recover. If prior information is used, the presentation of extreme stimulus values may be constrained so that the adaptive procedure estimates the true threshold. Watson and Pelli (1983), Harvey (1986), and Madigan and Williams (1987) discuss how prior information improves the threshold estimation from adaptive procedures. Reports of simulations should carefully describe how prior information is used, as this may cause differences in the biases found.

\section{Comparisons of Threshold Estimation of the Modified Best PEST With the Method of Constant Stimuli}

The simulations described above show that the modified Best PEST procedure does what it was intended to do: It uses an adaptive maximum likelihood approach to find any threshold along a perceiver's psychometric function, even for perceivers who have different PSEs and DLs. Before concluding, this paper considers a couple of claims made in the introduction. This section compares the efficiency of estimating thresholds from the modified Best PEST procedure with that of the more traditional method of constant stimuli.

When does the modified Best PEST procedure provide better estimates of perceivers' thresholds than the method of constant stimuli? To answer this question, simulations compared the threshold estimates from the modified Best PEST procedure and the method of constant stimuli. Comparisons were made in which the method of constant stimuli made different assumptions about the shapes of psychometric functions for perceivers. Different methods of constant stimuli assumed that the PSEs of perceivers could vary widely, or they could be fairly well known and assumed to be centralized.

To compare threshold estimates from the method of constant stimuli and Best PEST, both procedures are simulated with 72 trials. The threshold estimates from the method of constant stimuli are created by submitting all
72 stimuli and responses to logistic regression on SPSS, the same program which analyzed the data collected during Best PEST experimental sessions (although Finney, 1971, used Probit Analysis to determine thresholds when using the method of constant stimuli).

The results (see Tables 8A and 8B) show that the modified Best PEST generally provides more reliable estimates of thresholds than does the method of constant stimuli. However, the superiority of the adaptive method was less than might have been expected, given earlier reports comparing adaptive procedures (such as Pentland, 1980). Indeed, for certain combinations of perceiver's psychometric functions and distributions of stimuli presented, the method of constant stimuli yields estimates of the DL that are as reliable as those from adaptive methods. Still, adaptive methods are clearly superior, even if they do not provide dramatic improvements in the reliability of estimated thresholds.

\section{Comparing Threshold Estimates of Modified Best PEST With 2AFC}

This section compares threshold estimates from the modified Best PEST with estimates from 2AFC across two experimental situations. One situation is the simple discrimination of two similar signals. The second situation is the loudness comparison of different wavelength tones, which was described in the Introduction.

First, to measure the discriminability of two nearly similar tones, either $2 \mathrm{AFC}$ or the modified Best PEST can be used. In using $2 \mathrm{AFC}$, the experimenter presents the standard signal, and the standard plus delta signal on every trial, but the order is varied. The value of delta is increased when the perceiver makes an incorrect response and is decreased when the perceiver makes a correct response.

In the modified Best PEST, the experimenter presents the standard signal on the first interval, and then either a standard minus delta (smaller) or a standard plus delta (larger) signal on the second interval. Note that the delta signals are now identified as delta (smaller) or delta (larger) rather than just delta; these two deltas can have different values.

The comparisons are done under two situations. In one condition considered here, the two signals are similar, and we assume no constant error. That is, the first signal does not mask nor accentuate the second signal. The second condition is one in which there is constant error. The results are shown in Table 9.

The results show that the modified Best PEST provides more reliable estimates of the slope of the perceiver's psychometric function than does the $2 \mathrm{AFC}$ procedure, even when the perceiver has no constant error. When the perceiver has a constant error, the modified Best PEST is superior to the $2 \mathrm{AFC}$ task for providing more accurate and reliable estimates of the perceiver's thresholds, as well as indicating the presence of the perceiver's constant error.

The second experimental investigation describes the experiment discussed in the Introduction. In this situation. the experimenter studied the relative loudnesses of two 
Table 8A

Threshold Estimates From Best PEST

\begin{tabular}{|c|c|c|c|c|c|c|c|c|c|}
\hline \multirow{2}{*}{\multicolumn{2}{|c|}{$\begin{array}{l}\text { Simulated } \\
\text { Perceivers }\end{array}$}} & \multicolumn{4}{|c|}{$\begin{array}{l}\text { Estimated Thresholds } \\
\text { From Best PEST }\end{array}$} & \multicolumn{4}{|c|}{$\begin{array}{l}\text { Estimated Thresholds From } \\
\text { PEST and Regression }\end{array}$} \\
\hline & & \multicolumn{2}{|c|}{ PSE } & \multicolumn{2}{|c|}{ DL } & \multicolumn{2}{|c|}{ PSE } & \multicolumn{2}{|c|}{ DL } \\
\hline PSE & $\mathrm{DL}$ & $M$ & $S D$ & $M$ & $S D$ & $M$ & $S D$ & $M$ & $S D$ \\
\hline 0 & 3 & -0.05 & 1.27 & 3.22 & 1.23 & 0.00 & 0.91 & 2.82 & 0.71 \\
\hline 0 & 6 & 0.03 & 1.97 & 6.28 & 1.94 & 0.00 & 1.81 & 5.61 & 1.44 \\
\hline 0 & 12 & 0.20 & 3.36 & 12.08 & 3.44 & 0.24 & 3.37 & 10.94 & 3.11 \\
\hline 0 & 24 & 0.73 & 6.28 & 21.84 & 6.60 & 0.58 & 6.49 & 21.89 & 7.96 \\
\hline 12 & 3 & 12.05 & 1.54 & 3.23 & 1.30 & 12.03 & 0.91 & 2.84 & 0.72 \\
\hline 12 & 6 & 12.09 & 1.91 & 6.38 & 1.93 & 12.15 & 1.70 & 5.59 & 1.43 \\
\hline 12 & 12 & 11.87 & 3.39 & 12.24 & 3.22 & 12.02 & 3.40 & 11.04 & 3.11 \\
\hline 12 & 24 & 10.14 & 5.89 & 21.56 & 6.09 & $11.2 \mathrm{I}$ & 6.16 & 21.69 & 7.05 \\
\hline-24 & 3 & -24.09 & 1.25 & 3.40 & 1.37 & -24.05 & 0.93 & 2.89 & 0.72 \\
\hline-24 & 6 & -24.04 & 1.96 & 6.49 & 1.97 & -24.04 & 1.78 & 5.67 & 1.45 \\
\hline-24 & 12 & -23.70 & 3.34 & 12.26 & 3.24 & -23.99 & 3.42 & 11.10 & 3.12 \\
\hline-24 & 24 & -21.01 & 5.34 & 20.85 & 5.55 & -24.48 & 6.21 & 22.47 & 7.77 \\
\hline
\end{tabular}

Table 8B

Threshold Estimates From Constant Stimuli

\begin{tabular}{|c|c|c|c|c|c|c|c|c|c|c|c|c|c|}
\hline \multirow{2}{*}{\multicolumn{2}{|c|}{$\begin{array}{l}\text { Simulated } \\
\text { Perceivers }\end{array}$}} & \multicolumn{4}{|c|}{$\begin{array}{c}\text { Constant Stimuli } \\
\text { (9 levels) } \\
\text { Steep* }\end{array}$} & \multicolumn{4}{|c|}{$\begin{array}{c}\text { Constant Stimuli } \\
(9 \text { levels }) \\
\text { Shallow } \dagger\end{array}$} & \multicolumn{4}{|c|}{$\begin{array}{l}\text { Constant Stimuli } \\
\text { (17 levels) } \\
\text { Mixed } \neq\end{array}$} \\
\hline & & \multicolumn{2}{|c|}{ PSE } & \multicolumn{2}{|c|}{$\mathrm{DL}$} & \multicolumn{2}{|c|}{ PSE } & \multicolumn{2}{|c|}{$\mathrm{DL}$} & \multicolumn{2}{|c|}{ PSE } & \multicolumn{2}{|c|}{ DL } \\
\hline PSE & $\mathrm{DL}$ & $M$ & $S D$ & $M$ & $S D$ & $M$ & $S D$ & $M$ & $S D$ & $M$ & $S D$ & $M$ & $S D$ \\
\hline 0 & 3 & -0.05 & 1.31 & 1.84 & 1.40 & 0.17 & 1.57 & 2.68 & 1.00 & -0.09 & 1.94 & 2.38 & 1.37 \\
\hline 0 & 6 & 0.07 & 2.87 & 5.29 & 1.98 & 0.14 & 2.22 & 5.80 & 1.35 & 0.25 & 2.93 & 5.57 & 1.72 \\
\hline 0 & 12 & 0.21 & 4.39 & 11.43 & 2.80 & 0.19 & 3.43 & 12.29 & 3.58 & 0.44 & 4.21 & 11.84 & 2.64 \\
\hline 0 & 24 & 0.22 & 7.07 & 25.33 & 6.98 & 0.27 & 7.10 & 28.94 & 19.60 & 0.15 & 6.88 & 24.64 & 6.84 \\
\hline 12 & 3 & 11.87 & 1.74 & 1.98 & 1.48 & 11.86 & 1.43 & 2.60 & 1.03 & 12.03 & 1.83 & 2.25 & 1.39 \\
\hline 12 & 6 & 11.95 & 2.84 & 5.53 & 1.93 & 12.13 & 2.42 & 5.76 & 1.51 & 11,91 & 2.76 & 5.67 & 1.71 \\
\hline 12 & 12 & 12.12 & 4.44 & 11.42 & 2.73 & 12.21 & 4.00 & 12.27 & 3.76 & 11.61 & 4.25 & 11.61 & 2.86 \\
\hline 12 & 24 & 12.27 & 7.78 & 24.26 & 6.83 & 13.63 & 14.36 & 28.10 & 22.34 & 12.01 & 6.85 & 24.49 & 7.41 \\
\hline-24 & 3 & -23.97 & 1.55 & 1.96 & 1.46 & -24.28 & 2.41 & 2.55 & 1.72 & -24.15 & 1.96 & 2.51 & 1.33 \\
\hline-24 & 6 & -23.74 & 3.17 & 5.41 & 2.00 & -24.78 & 4.48 & 5.88 & 2.65 & -23.99 & 2.97 & 5.70 & 1.71 \\
\hline-24 & 12 & -24.05 & 4.55 & 11.60 & 3.31 & -25.74 & 9.79 & 2.52 & 5.71 & -24.30 & 4.64 & 11.76 & 2.96 \\
\hline-24 & 24 & -24.88 & 8.34 & 24.88 & 8.02 & -29.47 & 57.34 & 28.08 & 59.91 & -25.21 & 9.18 & 24.78 & 8.07 \\
\hline
\end{tabular}

Note-Number of trials/estimate is 72 . *Steep: Constant stimuli presented stimulus steps at $-4,-3,-2,-1,0,1,2,3$, and 4 logits (of the psychometric function assumed by Best PEST). Each stimulus step was presented eight times. 'Shallow: Constant stimuli presented stimulus steps at $-2,-1.5,-1,-0.5,0.0 .5,1.0,1.5$, and 2.0 logits (of the psychometric function assumed by Best PEST). Each stimulus step was presented eight times. $¥$ Mixed: Constant stimuli presented at stimulus steps $-4,-3.5,-3,-2.5,-2,-1.5,-1,-0.5,0,0.5,1,1.5,2,2.5,3,3.5$, and 4 (of the psychometric function assumed by Best PEST). Each stimulus step was presented four times, and $-2,-1,1$, and 2 were presented one extra time.

tones of different wavelengths. In this experiment, threshold estimation from the modified Best PEST is compared with threshold estimation using $2 \mathrm{AFC}$. The perceivers will be judging the loudnesses of two tones of different wavelengths (a $256-\mathrm{Hz}$ tone and a $440-\mathrm{Hz}$ tone). This question is difficult to address with the method of constant stimuli, because we would not know for a particular perceiver what intensity of a $256-\mathrm{Hz}$ tone is equal to the intensity of a $440-\mathrm{Hz}$ tone. If we look this up for an average perceiver in an equal-loudness chart, we find that a $256-\mathrm{Hz}$ tone of $60 \mathrm{~dB}$ generally equals the loudness of a $440-\mathrm{Hz}$ tone of $56 \mathrm{~dB}$ (Gelfand, 1981). If the experimenter assumes that the perceiver tested is average, the experimenter can run a $2 \mathrm{AFC}$ experiment with the standard signal specified as the $256-\mathrm{Hz}, 60-\mathrm{dB}$ signal, and then the standard plus delta signal can be the $440-\mathrm{Hz}$ signal, $56 \mathrm{~dB}$ plus some increment for the other signal. With this procedure, after the experimenter assumes the equivalence of two different signals, the experimenter can measure how well the perceiver discriminates these two signals.

If we run a modified Best PEST, we can determine the discriminability of two signals, we can experimentally determine the PSE for these signals, and we can measure constant error. To measure all of these characteristics, we will need to find four different thresholds: When the standard signal $(256 \mathrm{~Hz}, 60 \mathrm{~dB})$ is presented first, we need to estimate the intensity levels of the $440-\mathrm{Hz}$ signal that are judged to be 1 logit above and 1 logit below the standard. Then the standard signal $(256 \mathrm{~Hz}, 60 \mathrm{~dB})$ must be presented second, and the intensity of the $440-\mathrm{Hz}$ signal 
Table 9

Comparing Threshold Estimates From Modified Best PEST and 2AFC Task

\begin{tabular}{|c|c|c|c|c|c|c|c|c|c|c|c|}
\hline \multirow{2}{*}{\multicolumn{2}{|c|}{$\begin{array}{l}\text { Simulated } \\
\text { Perceiver } \\
\end{array}$}} & \multicolumn{4}{|c|}{$\begin{array}{l}\text { Estimated Thresholds } \\
\text { From Best PEST }\end{array}$} & \multicolumn{4}{|c|}{$\begin{array}{c}\text { Estimated Thresholds From } \\
\text { PEST and Regression }\end{array}$} & \multirow{2}{*}{\multicolumn{2}{|c|}{$\begin{array}{c}\begin{array}{c}\text { Estimated } \\
\text { Threshold } \\
\text { From } 2 \mathrm{AFC}\end{array} \\
\mathrm{DL} \\
\end{array}$}} \\
\hline & & \multicolumn{2}{|c|}{ PSE } & \multicolumn{2}{|c|}{ DL } & \multicolumn{2}{|c|}{ PSE } & \multicolumn{2}{|c|}{ DL } & & \\
\hline PSE & $\mathrm{DL}$ & $M$ & $S D$ & $M$ & $S D$ & $M$ & $S D$ & $M$ & $S D$ & $M$ & $S D$ \\
\hline 0 & 6 & 0.12 & 1.84 & 6.39 & 1.99 & 0.10 & 1.66 & 5.58 & 1.46 & 6.77 & 2.26 \\
\hline 0 & 12 & 0.18 & 3.28 & 12.25 & 3.52 & 0.18 & 3.25 & 11.25 & 3.41 & 13.38 & 3.85 \\
\hline 6 & 6 & 6.08 & 1.98 & 6.42 & 1.98 & 6.11 & 1.77 & 5.67 & 1.56 & 8.79 & 5.51 \\
\hline 6 & 12 & 5.91 & 3.19 & 12.23 & 3.37 & 5.99 & 3.21 & 11.12 & 3.35 & 14.05 & 3.59 \\
\hline
\end{tabular}

Table 10

Comparing Loudnesses of 256-Hz Signal to a 440-Hz Signal

\begin{tabular}{|c|c|c|c|c|c|c|c|c|c|c|}
\hline \multirow{2}{*}{\multicolumn{3}{|c|}{$\begin{array}{l}\text { Simulated } \\
\text { Perceiver }\end{array}$}} & \multicolumn{6}{|c|}{$\begin{array}{l}\text { Estimated Threshold } \\
\text { From Best PEST }\end{array}$} & \multirow{2}{*}{\multicolumn{2}{|c|}{$\begin{array}{r}\begin{array}{r}\text { Estimated } \\
\text { Threshold } \\
\text { From } 2 \mathrm{AFC}\end{array} \\
\mathrm{DL}\end{array}$}} \\
\hline & & & \multicolumn{2}{|c|}{ PSE } & \multicolumn{2}{|c|}{$\mathrm{DL}$} & \multicolumn{2}{|c|}{ ce } & & \\
\hline PSE & $\mathrm{DL}$ & ce & $M$ & $S D$ & $M$ & $S D$ & $M$ & $S D$ & $M$ & $S D$ \\
\hline 56 & 2 & 0 & 56.04 & 0.66 & 2.25 & 6.67 & 0.03 & 0.68 & 2.26 & 0.74 \\
\hline 56 & 4 & 0 & 56.05 & 1.12 & 4.24 & 1.15 & 0.01 & 1.13 & 4.44 & 1.17 \\
\hline 58 & 2 & 0 & 58.02 & 0.70 & 2.25 & 0.66 & 0.02 & 0.68 & 4.26 & 0.74 \\
\hline 58 & 4 & 0 & 58.11 & 1.10 & 4.19 & 1.15 & 0.01 & 1.11 & 6.52 & 1.20 \\
\hline 58 & 2 & 1 & 58.00 & 0.70 & 2.23 & 0.64 & 1.00 & 0.68 & 4.37 & 0.81 \\
\hline 58 & 4 & 1 & 57.97 & 1.15 & 4.19 & 1.08 & 0.98 & 1.07 & 6.55 & 1.20 \\
\hline
\end{tabular}

can be varied to find the intensity level that is 1 logit below the standard and 1 logit above the standard. The results are shown in Table 10.

The results show that the $2 \mathrm{AFC}$ procedure can estimate the discriminability of two signals nearly as well as the modified Best PEST when the PSE estimate between the two signals is accurate and the constant error is 0 . But when the PSE of the two signals differs or there is constant error, the modified Best PEST yields much more information about the perceiver's psychometric function. Depending on the experimental question, the modified Best PEST can provide much more information than can a $2 \mathrm{AFC}$ task.

\section{CONCLUSION}

Pentland's Best PEST procedure has been modified to estimate any threshold along a psychological continuum. In developing this procedure, a major concern was that the assumed logistic function would bias the estimates of perceivers' thresholds. Simulations described here, however, showed that this procedure closely estimated perceiver thresholds anywhere along the psychometric function.

Many simulations were completed to investigate how well this adaptive procedure worked. One finding of general importance is that the confidence interval around the estimated threshold is not sensitive to perceiver's sensory ability; rather, it depends on the assumptions and parameters determined by the experimenter. Another finding of general importance is that submitting trials from a Best PEST procedure to logistic regression yields more reliable threshold estimates than does using the direct threshold estimate from Best PEST. Another interesting simulation showed that the adaptive procedures were superior, but not dramatically so, to the method of constant stimuli.

In other simulations, the effect of implicit trials was looked at, the most efficient number of trials to run per block was investigated, and the efficiency of the modified Best PEST procedure and that of a $2 \mathrm{AFC}$ procedure were compared. All of these findings can be used by experimenters using maximum likelihood procedures to estimate thresholds.

As a final endorsement, we (Keele \& Ivry, 1987; Keele, Pokorny, Corcos, \& Ivry, 1985) have found this modified Best PEST procedure efficient when obtaining threshold estimates with relatively few trials in individual difference studies, when the reliability of each subject's data is critical.

\section{REFERENCES}

EMERSON, P. L. (1984). Observations on a maximum likelihood method of sequential threshold estimation and a simplified approximation. Perception \& Psychophysics, 36, 199-203.

EMERSON, P. L. (1986). Observations on maximum-likelihood and Bayesian methods of forced-choice sequential threshold estimation. Perception \& Psychophysics, 39, 151-153.

ENGEN, T. (1971). Psychophysics 1. Discrimination and Detection. In J. W. Kling \& L. A. Riggs (Eds.), Woodworth and Schlosberg's experimental psychology (pp. 11-46). New York: Holt, Rinehart, \& Winston.

Finney, D. J. (1971). Probit analysis (3rd ed.). Cambridge: Cambridge University Press.

Gelfand, S. A. (1981). Hearing: An introduction to psychological and physiological acoustics. New York: Dekker.

GREEN, D. M. (1991). A maximum-likelihood method for estimating thresholds in a yes-no task. Journal of the Acoustical Society of America, 93, 2096-2105.

HaRVEY, L. O., JR. (1986). Efficient estimation of sensory thresholds. Behavior Research Methods. Instruments, \& Computers, 18, 623-632. 
Keele, S. W., \& IVRY, R. I. (1987). Modular analysis of timing in motor skill. Psychology of Learning \& Motivation, 21, 183-228.

Keele, S. W., Pokorny, R. A., Corcos, D. M., \& Ivry, R. I. (1985). Do perception and motor production share common timing mechanisms: A correlational analysis. Acta Psychologia, 60, 173-191.

King-Smith, P. E., Grigsby, S. S., Vingris, A. J., Benes, S. C., \& SupowiT, A. (1994). Efficient and unbiased modifications of the QUEST threshold method: Theory, simulations, experimental evaluation and practical implementation. Vision Research, 34, 885-912.

Lieberman, H. R., \& Pentland, A. P. (1982). Microcomputer-based estimation of psychophysical thresholds: The Best PEST. Behavior Research Methods \& Instrumentation, 14, 21-25.

MacMillan, N. A., \& Creelman, C. D. (1991). Detection theory: A user's guide. New York: Cambridge University Press.

Madigan, R., \& Williams, D. (1987). Maximum-likelihood psychometric procedures in two-alternative forced-choice: Evaluations and recommendations. Perception \& Psychophysics, 42, 240-249.
Pentland, A. (1980). Maximum likelihood estimation: The best PEST. Perception \& Psychophysics, 28, 377-379.

Shelton, B. R. (1983). Rapid calculation procedures for the maximumlikelihood method of adaptive psychophysics. Behavior Research Methods \& Instrumentation, 15, 87-88.

Simpson, W. A. (1989). The step method: A new adaptive psychophysical procedure. Perception \& Psychophysics, 45, 572-576.

TAYLOR, M. M., \& CREELMAN, D. C. (1967). PEST: Efficient estimates on probability functions. Journal of the Acoustical Society of America, 41, 782-787.

TAYlor, M. M., Forbes, S. M., \& Creelman, C. D. (1983). PEST reduces bias in forced choice psychophysics. Journal of the Acoustical Society of America, 74, 1367-1374.

Treutwein, B. (1995). Adaptive psychophysical procedures. Vision Research, 35, 2503-2522.

Watson, A. B., \& Pelli, D. G. (1983). QUEST: A Bayesian adaptive psychometric method. Perception \& Psychophysics, 33, 113-120.

\section{LISTING \\ The Modified Best PEST Procedure, Implemented in PASCAL}

Note--This program finds one threshold anywhere along the perceiver's psychometric func-
tion. In most of the simulations, two thresholds were found by a slightly more elaborate program. But the essence of that program is here.

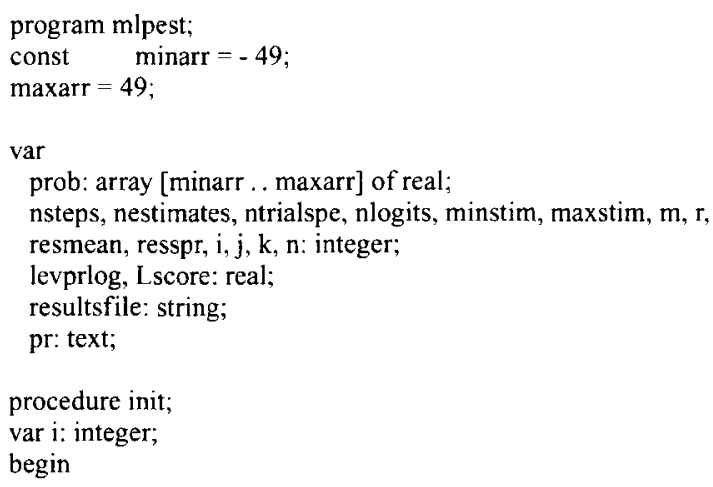

write(' $\$$ of stimulus steps presentable= '); readln (nsteps); write('\# of estimates of the threshold= '); readin (nestimates); write('\# of trials per estimate= '); readln (ntrialspe); write('\# of assumed logits in stim range = '); readln (nlogits); write('target threshold sought in L units= '); readln (Lscore); maxstim := nsteps div 2;

minstim :=-maxstim; levprlog $:=($ nsteps -1$) /$ nlogits; write('write file name $=$ '); readln (resultsfile); assign(pr,resultsfile);

rewrite(pr);

write('sim res mean='); readln (resmean); write('sim res spr= '); readin (resspr); writeln(pr, 'minstim maxstim lev/logits Lscore, res mean, res spr); writeln(pr,minstim:5,maxstim:9, levprlog: 12:2, Lscore:9:2, resmean:8, resspr:9), end;

procedure thold;

var max: real;

$\mathrm{i}, \mathrm{pl}, \mathrm{p} 2$ : integer;

begin

$\max :=-1000$

for $\mathrm{i}:=$ minstim to maxstim do

prob $[\mathrm{i}]:=\operatorname{prob}[\mathrm{i}]+\ln (1 /(1+\exp ((-\mathrm{r}) *((\mathrm{~m}-\mathrm{i}+$ Lscore*levprlog $) / \operatorname{levpr} \log ))))$;

("next, find $\mathrm{m}$. the most probable stimulus step; even check for ties *) 


\section{LISTING (Continued)}

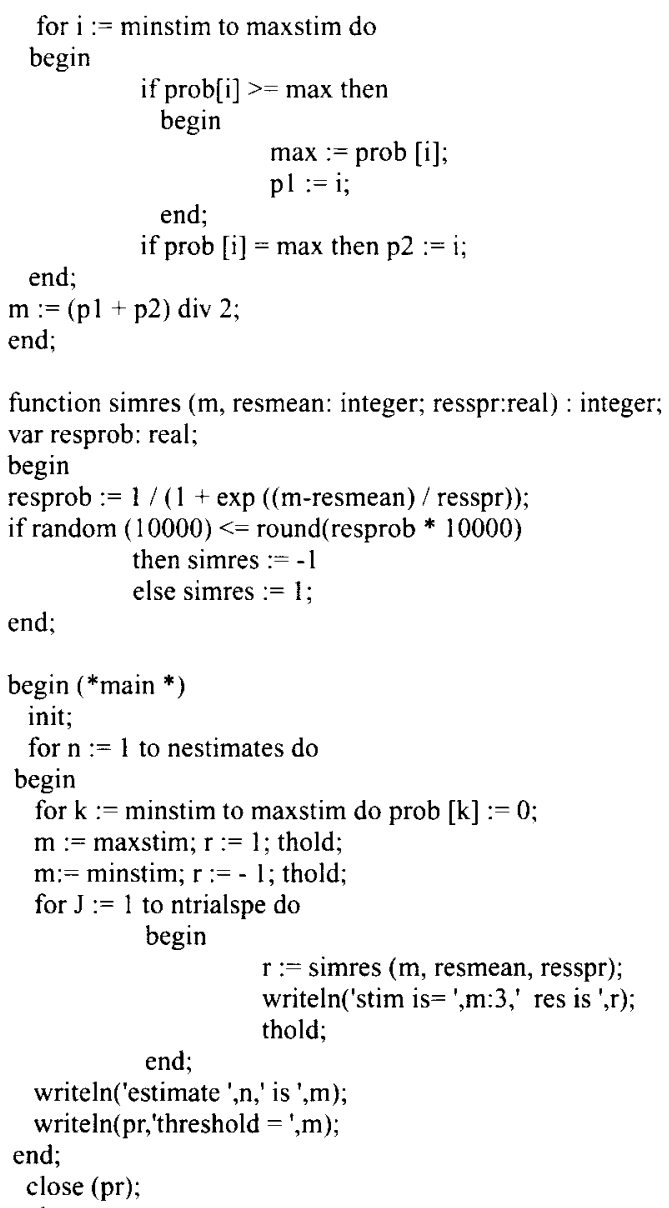

1. The PASCAL variable "prob" is the threshold probability array, which stores the probabilities that the corresponding stimulus level is the threshold being sought.

2. The multiplication of the prob array by the adjusting logistic ogive is implemented with a $\log$ transformation of the logistic ogive. This eliminates the possibility of numbers that are too small to compute, eliminating this possible computer error.

3. Implementing the multiplication of the threshold probability array by the adjusting logistic ogive uses the same variable names as suggested in the "in principle" implementation in the text, except that the "s" in-principle variable is replaced with "levels-per-logit," or "levprlog".

4. The perceiver is simulated with the simres function: It returns -1 for "less than" and +1 for "greater than." If a random number generated (by the PASCAL call 'random') is less than the perceived signal strength — given $\mathrm{m}$, the stimulus level presented; resmean, the midpoint of the perceiver's psychometric function; and resspr, the slope (or spread) of the perceiver's psychometric function-simres returns "less than."

5. The Best PEST assumes that the perceiver's psychometric function has a slope specified by the number of presentable (nsteps) divided by how many logits are assumed to be found across the stimulus steps (nlogits). The levprlog variable is the number of stimulus steps (or levels) per each logit of the perceiver assumed by Best PEST. Note that we have two slopes of psychometric functions: one is levprlog, which PEST assumes of the perceiver. Then we also have the perceiver's true slope, specified in resspr. 